\title{
Analytical Evaluation of the Neutrino-Nucleon Interaction Cross-Sections at Ultrahigh Energy
}

\author{
Pijush Kanti Dhar ${ }^{1}$ and D. K. Choudhury ${ }^{2}$ \\ ${ }^{1}$ Department of Physics, Arya Vidyapeeth College, Guwahati 781016, India \\ ${ }^{2}$ Department of Physics, Gauhati University, Guwahati 781014, India
}

Correspondence should be addressed to Pijush Kanti Dhar; dr_pijush@yahoo.co.in

Received 2 November 2013; Accepted 20 November 2013; Published 2 February 2014

Academic Editors: K. Cho, I. Lokhtin, and S. Paganis

Copyright ( 2014 P. K. Dhar and D. K. Choudhury. This is an open access article distributed under the Creative Commons Attribution License, which permits unrestricted use, distribution, and reproduction in any medium, provided the original work is properly cited.

\begin{abstract}
The ultrahigh energy (UHE) neutrino-nucleon interaction cross-sections are evaluated analytically in one loop by using the solutions of Dokshitzer-Gribov-Lipatov-Altarelli-Parisi (DGLAP) equations. Since the genesis of the UHE neutrinos is various sources which have energy more than $10^{5} \mathrm{GeV}$, so parton distributions are extrapolated at ultralow $x$ to determine the charged current and the neutral current cross-sections. We then compare our analytically obtained extrapolated results with the next-toleading order (NLO) results of various other authors. A good agreement between the two reflects the veracity of our approach of extrapolation at ultralow $x$.
\end{abstract}

\section{Introduction}

With the help of analytical solutions of the nonsinglet and the singlet DGLAP equation [1], we will determine analytically the UHE neutrino-nucleon interaction cross-sections both for the charged and the neutral current in the leading order (LO). We will then compare our predictions with the results of several other authors [2-4] and study the range of validity of our approach. In Section 2, we develop the essential formalism while, in Section 3, we put forward the results. Comments and conclusions are given in Section 4.

\section{Formalism}

2.1. Charged Current-Analytical Solution. The differential cross-section for the charged current (CC) UHE neutrinonucleon interaction is given by [5]

$$
\frac{d^{2} \sigma_{\mathrm{CC}}^{\gamma(\bar{v}) N}}{d x d Q^{2}}=\frac{G_{F}^{2}}{2 \pi}\left(1+\frac{Q^{2}}{M_{W}^{2}}\right)^{-2}
$$

$$
\begin{aligned}
& \times \frac{1}{x}[\left(1-y+\frac{y^{2}}{2}\right) F_{2}^{\gamma(\bar{\nu})}(x, t) \\
&\left. \pm y\left(1-\frac{y}{2}\right) x F_{3}^{\nu(\bar{\nu})^{\mathrm{CC}}}(x, t)\right],
\end{aligned}
$$

where Fermi coupling constant $G_{F}=1.1663 \times 10^{-5} \mathrm{GeV}^{-2}$, $Q^{2}=x y s, s=2 M E_{\gamma(\bar{v})}$ is the centre-of-mass energy squared, $x$ is the usual Bjorken variable, and $y$ is the inelasticity parameter. The total cross-section for UHE CC interaction in an isoscalar target $N[\equiv(p+n) / 2]$ is given by

$$
\sigma_{\mathrm{CC}}^{\nu(\bar{\gamma}) N}=\int_{Q^{2} / s}^{1} d x \int_{\mathrm{Q}_{0}^{2}}^{s} d Q^{2} \frac{d^{2} \sigma_{\mathrm{CC}}^{\nu(\bar{v})}}{d x d Q^{2}} .
$$

By solving the DGLAP equations [1] analytically, one gets the expressions for the nonsinglet structure function $F^{\mathrm{NS}}(x, t)$ and the singlet structure function $F_{2}^{S}(x, t)$ as $[6,7]$

$$
F^{\mathrm{NS}}(x, t)=F^{\mathrm{NS}}\left(x, t_{0}\right)\left(\frac{t}{t_{0}}\right)^{n(x, t)}, \quad\left(n(1, t)>0, t \geq t_{0}\right),
$$




$$
\begin{array}{r}
F_{2}^{S}(x, t)=F_{2}^{S}\left(x, t_{0}\right) \frac{t^{k(x, t)}}{t_{0}^{k\left(x, t_{0}\right)}} \frac{\left[X^{S}(x)\right]^{k(x, t)}}{\left[X^{S}(x)\right]^{k\left(x, t_{0}\right)}}, \\
\left(K(1, t)>0, t \geq t_{0}\right),
\end{array}
$$

where $t=\log \left(Q^{2} / \Lambda^{2}\right)$ and $t_{0}=\log \left(Q_{0}^{2} / \Lambda^{2}\right), Q^{2}\left(\equiv-q^{2}\right)$ is the four-momentum transfer, and $\Lambda$ is the QCD cut-off parameter. Here, $k(x, t)$ is the same as $H_{\text {expt }}^{S}(x, t)$ given in [7]; namely,

$$
\begin{aligned}
H^{S}(x, t)= & 1.284[-4.64 x+1.02(1-x)] \\
& \times\left(1.52-1.16 s-0.06 s^{2}\right),
\end{aligned}
$$

where

$$
s \equiv \log \left[\frac{\log \left[Q^{2} /(0.220 \mathrm{GeV})^{2}\right]}{\log \left[Q_{0}^{2} /(0.220 \mathrm{GeV})^{2}\right]}\right] .
$$

And $Q_{0}^{2}=1 \mathrm{GeV}^{2}$ as per MRST f4 2004 distribution. On the other hand, $n(x, t)$ is the same as $H(x, t)$ given in [6]; namely,

$$
H(x, t)=0.380[-5.796 x+0.996(1-x)],
$$

which is found to be independent of $Q^{2}$. Again, $X^{S}(x)$ is given by [6]

$$
X^{S}(x)=\exp \left[-\int \frac{d x}{P_{1}(x)}\right]
$$

where

$$
P_{1}(x, t)=-A_{f} x\left[2 \log \left(\frac{1}{x}\right)+\left(1-x^{2}\right)\right] .
$$

Now with the help of (3) and (4), (1) can be transformed as

$$
\begin{aligned}
\frac{d^{2} \sigma_{\mathrm{CC}}^{\nu(\bar{\nu}) N}}{d x d Q^{2}}=\frac{G_{F}^{2}}{2 \pi}(1 & \left.+\frac{Q^{2}}{M_{W}^{2}}\right)^{-2} \\
\times \frac{1}{x}[ & \left(1-y+\frac{y^{2}}{2}\right) F_{2}^{\nu(\bar{\nu})^{\mathrm{CC}}}\left(x, t_{0}\right)\left(\frac{t}{t_{0}}\right)^{k(x, t)} \\
& \times \frac{\left[X^{S}(x)\right]^{k(x, t)}}{\left[X^{S}(x)\right]^{k\left(x, t_{0}\right)} \pm y\left(1-\frac{y}{2}\right) x} \\
& \left.\times F_{3}^{\nu(\bar{\nu})^{\mathrm{CC}}}\left(x, t_{0}\right)\left(\frac{t}{t_{0}}\right)^{n(x, t)}\right] .
\end{aligned}
$$

We will now consider light quarks only and neglect heavy quarks. For charged current (CC) interactions, the standard expressions for structure functions in leading order are given by [5]

$$
\begin{gathered}
F_{1}^{\nu, \text { light }}=\frac{1}{2}(\bar{u}+\bar{d})+\frac{1}{2}(d+u)\left|V_{u d}\right|^{2}+s\left|V_{u s}\right|^{2}, \\
F_{2}^{\nu, \text { light }}=2 x F_{1}^{\nu, \text { light }}, \\
F_{3}^{\nu, \text { light }}=-(\bar{u}+\bar{d})+(d+u)\left|V_{u d}\right|^{2}+2 s\left|V_{u s}\right|^{2},
\end{gathered}
$$

where $u=u\left(x, Q^{2}\right)$ and so forth and $V_{u d}, V_{u s}$, and so forth are the relevant Cabibbo-Kobayashi-Maskawa (CKM) matrix elements $[8,9]$. Now, putting the explicit parton distributions from (11) in (10) and then using (2), one gets the expression for total cross-section for the charged current [10]:

$\sigma_{\mathrm{CC}}^{\nu(\bar{\nu}) N}$

$$
\begin{aligned}
=\frac{G_{F}^{2}}{2 \pi} \int_{Q_{0}^{2}}^{s} d Q^{2} & \left(1+\frac{Q^{2}}{M_{W}^{2}}\right)^{-2} \\
\times \int_{Q^{2} / s}^{1} \frac{d x}{x}[ & \left(1-\frac{Q^{2}}{x s}+\frac{Q^{4}}{2 x^{2} s^{2}}\right) \\
& \times 2 x\left\{\frac{1}{2}\left[\bar{u}\left(x, t_{0}\right)+\bar{d}\left(x, t_{0}\right)\right]\right. \\
& +\frac{1}{2}\left[d\left(x, t_{0}\right)+u\left(x, t_{0}\right)\right]\left|V_{u d}\right|^{2} \\
& +\frac{Q^{2}}{x s}\left(1-\frac{Q^{2}}{2 x s}\right) x \\
& \times\left\{-\left[\bar{u}\left(x, t_{0}\right)+\bar{d}\left(x, t_{0}\right)\right] \times \frac{t^{k(x, t)}}{t_{0}^{k\left(x, t_{0}\right)}} \frac{\left[X^{S}(x)\right]^{k(x, t)}}{\left[X^{S}(x)\right]^{k\left(x, t_{0}\right)}}\right. \\
& \left.+\left[d\left(x, t_{0}\right)+u\left(x, t_{0}\right)\right]\left|V_{u d}\right|^{2}+2 s\left|V_{u s}\right|^{2}\right\} \\
& \left.\times\left(\frac{t}{t_{0}}\right)^{n(x, t)}\right] \cdot
\end{aligned}
$$

2.2. Neutral Current-Analytical Solution. For UHE neutral current interaction, one has the following expression for total cross-section:

$$
\sigma_{\mathrm{NC}}^{\nu(\bar{v}) N}=\int_{Q^{2} / s}^{1} d x \int_{\mathrm{Q}_{0}^{2}}^{s} d Q^{2} \frac{d^{2} \sigma_{\mathrm{NC}}^{\nu(\bar{v}) N}}{d x d Q^{2}}
$$

where

$$
\begin{aligned}
\frac{d^{2} \sigma_{\mathrm{NC}}^{\nu(\bar{\nu}) N}}{d x d Q^{2}}=\frac{G_{F}^{2}}{2 \pi}\left(1+\frac{Q^{2}}{M_{Z}^{2}}\right)^{-2} \frac{1}{x} & {\left[\left(1-y+\frac{y^{2}}{2}\right) F_{2}^{\nu(\bar{\nu})^{\mathrm{NC}}}(x, t)\right.} \\
& \left. \pm y\left(1-\frac{y}{2}\right) x F_{3}^{\nu(\bar{\nu})^{\mathrm{NC}}}(x, t)\right] .
\end{aligned}
$$

These two equations are to be compared with (2) and (1), respectively, of the charged current. Putting the LO expressions for structure functions involving neutral currents (NC) [5] for light quarks in (14) and with the help of (13), one gets 
the expression for total cross-section for the neutral current as [10]

$$
\begin{aligned}
& \sigma_{\mathrm{NC}}^{\nu(\bar{v}) N} \\
& =\frac{G_{F}^{2}}{2 \pi} \int_{Q_{0}^{2}}^{s} d Q^{2}\left(1+\frac{Q^{2}}{M_{Z}^{2}}\right)^{-2} \\
& \times \int_{Q^{2} / s}^{1} \frac{d x}{x}\left[\left(1-\frac{Q^{2}}{x s}+\frac{Q^{4}}{2 x^{2} s^{2}}\right)\right. \\
& \times\left\{0.1432 x \times\left[u\left(x, t_{0}\right)+\bar{u}\left(x, t_{0}\right)+d\left(x, t_{0}\right)\right.\right. \\
& \left.+\bar{d}\left(x, t_{0}\right)\right]+0.1849 x \\
& \times\left[u\left(x, t_{0}\right)+\bar{u}\left(x, t_{0}\right)+d\left(x, t_{0}\right)\right. \\
& \left.\left.+\bar{d}\left(x, t_{0}\right)+4 s\right]\right\} \frac{t^{k(x, t)}}{t_{0}^{k\left(x, t_{0}\right)}} \frac{\left[X^{S}(x)\right]^{k(x, t)}}{\left[X^{S}(x)\right]^{k\left(x, t_{0}\right)}} \\
& \pm \frac{Q^{2}}{x s}\left(1-\frac{Q^{2}}{2 x s}\right) \\
& \times\left\{0 . 2 6 8 x \left[u\left(x, t_{0}\right)-\bar{u}\left(x, t_{0}\right)+d\left(x, t_{0}\right)\right.\right. \\
& \left.\left.\left.-\bar{d}\left(x, t_{0}\right)\right]\right\} \times\left(\frac{t}{t_{0}}\right)^{n(x, t)}\right] .
\end{aligned}
$$

2.3. Extrapolation at Ultralow $x$. In the ultrahigh energy domain of the neutrino $\left(E_{v}>10^{5}\right)$, all perturbative calculations have to be done at ultralow Bjorken $x\left(x<10^{-5}\right)$. Particularly, the sensitivity of UHE neutrino interactions is greatest in the domain $x \sim 10^{-8}$ and $Q^{2} \sim M_{W}^{2}$. Deep inelastic scattering (DIS) data at HERA [11] is valid only for $(x \geq$ $\left.10^{-5}\right)$. The greatest available energy $\sqrt{s_{e p}}$ available at HERA is $314 \mathrm{GeV}$, which is far below the expected UHE neutrinonucleon collision energies of about $\sqrt{s_{\nu N}}=10^{6} \mathrm{GeV}[5,12]$. So it is not possible to scan the ultrahigh energy region and evaluate the UHE cross-section from the present DIS experiments alone.

To determine the cross-sections for UHE neutrinonucleon interaction numerically, one way is to parametrize the parton distribution functions by global fitting to a rich set of available data at low values of $Q^{2}$ ( say $Q^{2}=Q_{0}^{2}$ ). The numerical solutions of the DGLAP equations [1] are then used to evolve parton densities at higher values of $Q^{2}$ $\left(Q^{2}>Q_{0}^{2}\right)$. This $Q^{2}$-evolution of small-x parton distribution is not the right choice to scan the region of UHE neutrinonucleon interactions as the evaluation is valid in the region of $x>10^{-5}$. Extensive extrapolation [2, 3, 13] is then carried out to determine the parton densities from the $\left(x, Q^{2}\right)$ domain of terrestrial accelerator HERA to the ultrasmall values of Bjorken $x$, where there is no data. But unfortunately, this type of extrapolation brings about some uncertainties in the estimated UHE neutrino-nucleon cross-sections [5] measured with the help of neutrino telescopes. This is because we are not so sure about what is happening in the region $x \rightarrow$ 0 and our ignorance forces us to take different assumptions (or educated guesses) about the behaviour of the distribution functions at ultrasmall $x$, leading to the large variations of different evaluated cross-sections.

In our analytical process of determining UHE neutrinonucleon cross-section, we adopt the process of extrapolating parton densities at ultralow Bjorken $x\left(x<10^{-5}\right)$, which will allow us to obtain reasonable cross-section in the region of $x$ which has not been explored by any existing terrestrial DIS experiment. Since the most dominant contribution to crosssection comes from singlet structure function $F_{2}$, so one can safely neglect the contribution from nonsinglet structure function $x F_{3}$. Since the sudden booming up in the production of sea quarks and gluons at ultralow $x$ is contained in the factor $\log (1 / x)$, so for carrying out extrapolation, we multiply $F_{2}(x, t)$ by the factor $\log (1 / x)$ in (1) and (14), leading to the generation of the following two modified extrapolated equations for the charged and the neutral current in place of nonextrapolated (12) and (15), respectively:

$$
\begin{aligned}
& \sigma_{\mathrm{CC}}^{\nu(\bar{\nu}) N} \\
& =\frac{G_{F}^{2}}{2 \pi} \int_{Q_{0}^{2}}^{s} d Q^{2}\left(1+\frac{Q^{2}}{M_{W}^{2}}\right)^{-2} \\
& \times \int_{Q^{2} / s}^{1} \frac{\log (1 / x)}{x}\left[\left(1-\frac{Q^{2}}{x s}+\frac{Q^{4}}{2 x^{2} s^{2}}\right)\right. \\
& \times 2 x\left\{\frac{1}{2}\left[\bar{u}\left(x, t_{0}\right)+\bar{d}\left(x, t_{0}\right)\right]\right. \\
& +\frac{1}{2}\left[d\left(x, t_{0}\right)+u\left(x, t_{0}\right)\right] \\
& \left.\times\left|V_{u d}\right|^{2}+s\left|V_{u s}\right|^{2}\right\} \\
& \left.\times \frac{t^{k(x, t)}}{t_{0}^{k\left(x, t_{0}\right)}} \frac{\left[X^{S}(x)\right]^{k(x, t)}}{\left[X^{S}(x)\right]^{k\left(x, t_{0}\right)}}\right] d x, \\
& \sigma_{\mathrm{NC}}^{\nu(\bar{v}) N} \\
& =\frac{G_{F}^{2}}{2 \pi} \int_{Q_{0}^{2}}^{s} d Q^{2}\left(1+\frac{Q^{2}}{M_{Z}^{2}}\right)^{-2} \\
& \times \int_{Q^{2} / s}^{1} \frac{\log (1 / x)}{x} \\
& \times\left[\left(1-\frac{Q^{2}}{x s}+\frac{Q^{4}}{2 x^{2} s^{2}}\right)\right. \\
& \times\left\{0.1432 x \times\left[u\left(x, t_{0}\right)+\bar{u}\left(x, t_{0}\right)\right.\right. \\
& \left.+d\left(x, t_{0}\right)+\bar{d}\left(x, t_{0}\right)\right] \\
& +0.1849 x \times\left[u\left(x, t_{0}\right)+\bar{u}\left(x, t_{0}\right)\right. \\
& \left.\left.+d\left(x, t_{0}\right)+\bar{d}\left(x, t_{0}\right)+4 s\right]\right\} \\
& \left.\times \frac{t^{k(x, t)}}{t_{0}^{k\left(x, t_{0}\right)}} \frac{\left[X^{S}(x)\right]^{k(x, t)}}{\left[X^{S}(x)\right]^{k\left(x, t_{0}\right)}}\right] d x \text {. }
\end{aligned}
$$




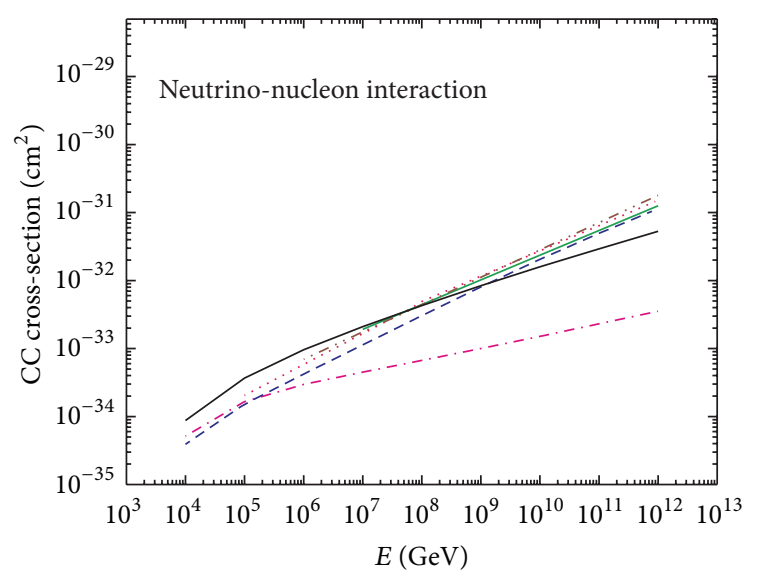

... Our analytical result without extrapolation

- - - Our numerical result (GRV94)

...... Amanda Cooper Sarkar (ZEUS-S global PDF)

— Gandhi et al. (CTEQ4)

Connolly et al. (MSTW)

_ Our analytical result with extrapolation

FIGURE 1: Our analytically obtained cross-section $\sigma_{\mathrm{CC}}^{\nu N}\left(\mathrm{~cm}^{2}\right)$ is compared with results of other authors [2-4] and our previous numerical result [10].

\section{Results and Discussions}

By using MRST 2004 f4 LO input parton distributions at $Q^{2}=$ $\mu_{\mathrm{LO}}^{2}=1 \mathrm{GeV}^{2}[14,15]$, we estimate the values of extrapolated cross-sections for $\nu N$ interactions for the charged and the neutral current from (16). This has to be compared with our nonextrapolated analytical results obtained earlier [10]. It is pertinent to note that the expression of the exponent $k(x, t)$ that has to be used in (16) has to be taken from (5), which was obtained in [7] by using MRST $2004 \mathrm{f} 4 \mathrm{LO}$ input parton distributions. The use of any input parton distributions other than MRST $2004 \mathrm{f} 4$ would change the expression of $k(x, t)$. That is why we have used here exclusively MRST 2004 f4 LO input parton distributions for finding out values of extrapolated cross-sections.

In Figures 1 and 2, our analytical extrapolated values of cross-section for the charged and the neutral current, that is, $\sigma_{\mathrm{CC}}^{\nu N}\left(\mathrm{~cm}^{2}\right)$ and $\sigma_{\mathrm{NC}}^{\nu N}\left(\mathrm{~cm}^{2}\right)$, respectively, are plotted against neutrino energy. Next, we also plot numerically obtained NLO results of various other authors [2-4] in the same figures. We also plot our nonextrapolated analytical results and nonextrapolated numerical results obtained earlier [10]. At low energies, both of our analytical nonextrapolated crosssections obtained earlier [10] and analytical extrapolated cross-sections obtained from (16) rise sharply. In this region, the cross-section is directly proportional to the energy of the neutrinos, where the propagator effect to the cross-section is totally absent [2]. For $E_{v} \geq 10^{3} \mathrm{GeV}$ and specially for $E_{v}$ exceeding $10^{4} \mathrm{GeV}$, there is a power suppression from the boson propagator, coupled with a logarithmic growth of the parton distribution functions (PDFs). But the dampening effect due to the propagator overpowers the logarithmic

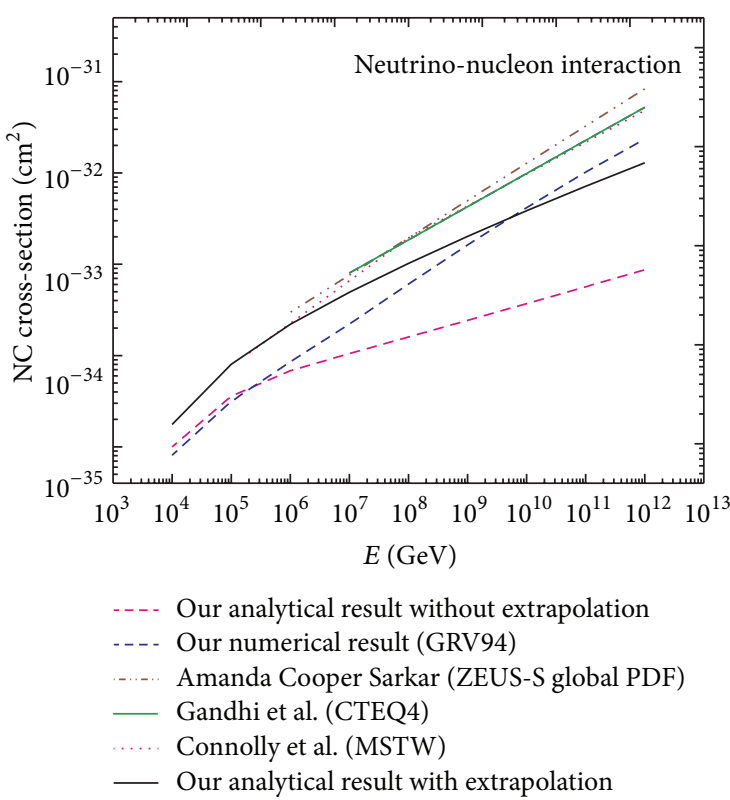

FIGURE 2: Our analytically obtained cross-section $\sigma_{\mathrm{NC}}^{\nu N}\left(\mathrm{~cm}^{2}\right)$ is compared with results of other authors [2-4] and our previous numerical result [10].

growth of the PDFs, creating dampening of the total crosssection [16].

As clearly evident from Figures 1 and 2, for neutrino energies exceeding $10^{4} \mathrm{GeV}$, due to excessive dampening, our previously calculated nonextrapolated analytically obtained values of cross-section [10] fall far short of the numerically obtained values of cross-section obtained by various other authors [2-4] as well as our previously obtained numerical results [10]. On the other hand, our currently obtained extrapolated analytical values of cross-section are more or less comparable with the NLO results of those authors in that region.

For $10^{4}<E_{v}<10^{8} \mathrm{GeV}$, our analytically obtained extrapolated results for charged current are a little above the numerically obtained NLO results of other authors [2-4], as can be seen in Figure 1. In between $E_{v}=10^{8} \mathrm{GeV}$ and $E_{v}=10^{9} \mathrm{GeV}$, our extrapolated results for charged current exactly coincide with the results of those authors. At higher energies up to $E_{v}=10^{12} \mathrm{GeV}$, our extrapolated results get a little dampened in comparison to the results of those authors.

It is evident from Figure 2 that our analytically obtained extrapolated results for neutral current are slightly dampened in comparison to the numerically obtained NLO results of those authors [2-4] all throughout the energy range of the UHE neutrino, with deviation becoming greatest at $10^{12} \mathrm{GeV}$. Of course, our extrapolated results obtained in this paper coincide nicely with our previously obtained numerical results [10] around $E_{v}=10^{10} \mathrm{GeV}$.

For high energy range $10^{8} \leq E_{v} \leq 10^{12}$, the dampening in our extrapolated results for both charged and neutral current is due to our neglect of the effect of heavy quarks in 
parton distribution functions (PDFs). The inclusion of heavy quark effect is expected to minimize the gap between our analytical extrapolated results and the numerical results of other authors in that range.

The origin of the difference between our results and results of other authors (presented in Figures 1 and 2) is because of the following factors.

(1) We have neglected the finite $x$ corrections coming from the higher derivatives of $\partial F_{2}^{S}(x, t) / \partial x$ and $\partial G(x, t) / \partial x$ in Taylor approximation of DGLAP equations in case of $t$-evolution of $F_{2}^{S}(x, t)$ at one loop level in [7] (4). This expression for $F_{2}^{S}(x, t)$ had been used while deriving (16) and so certain approximations have been introduced while deriving our analytically derived extrapolated results.

(2) We have done calculations at leading order involving LO parton distributions, whereas the calculations done by other authors at next-to-leading order are based on NLO parton distributions.

(3) We have done our calculations analytically, whereas other authors have done their calculations numerically. Obviously numerical approaches are more precise than analytical approaches. Different theoretical approaches will obviously bring certain amount of variations in the determination of values of crosssection.

(4) There might be a small variation of about $2 \%$ to $3 \%$ just due to different PDF choices up to $E_{v}=10^{7} \mathrm{GeV}$, but above that energy, specially for energy around $E_{v}=10^{12} \mathrm{GeV}$, the variations may reach up to more than $15 \%$.

(5) We have not taken into consideration the effect of heavy quarks, whose effect becomes pronounced around high energy, particularly around $10^{12} \mathrm{GeV}$.

The uncertainty in determining the cross-sections depends only in modest way on the choice of standard parton distribution functions up to energy $E_{v}=10^{7} \mathrm{GeV}$. It was found that, for $E_{v}>10^{7} \mathrm{GeV}$, predictions of crosssections depend on the untested various assumptions about the behaviour of parton distribution functions around the region $x \rightarrow 0[2,5]$ and the resulting uncertainty reaches typically a factor $2^{ \pm 1}$ around $10^{11} \mathrm{GeV}[5]$.

\section{Comments and Conclusions}

In conclusion, it is pertinent to note that our analytically extrapolated result tallies reasonably with the results of other authors [2-4] all throughout the energy spectrum of the UHE neutrino. The process of extrapolation of the parton densities at ultralow $x$ becomes a strong tool for us for bringing our earlier nonextrapolated analytical results [10] very close to the numerically obtained NLO results of those authors. It is expected that contribution due to heavy quarks will improve the result further in the top region of the UHE neutrino spectrum, specially around $10^{12} \mathrm{GeV}$.

\section{Conflict of Interests}

The authors declare that there is no conflict of interests regarding the publication of this paper.

\section{References}

[1] G. Altarelli and G. Parisi, "Asymptotic freedom in parton language," Nuclear Physics B, vol. 126, no. 2, pp. 298-318, 1977.

[2] R. Gandhi, C. Quigg, M. Reno, and I. Sarcevic, "Neutrino interactions at ultrahigh energies," Physical Review D, vol. 58, no. 9, Article ID 093009, 15 pages, 1998.

[3] A. Connolly, S. Robert Thorne, and D. Waters, "Calculation of high energy neutrino-nucleon cross sections and uncertainties using the MSTW parton distribution functions and implications for future experiments," Physical Review D, vol. 83, no. 11, Article ID 113009, 13 pages, 2011.

[4] A. Cooper-Sarkar and S. Sarkar, "Predictions for high energy neutrino cross-sections from the ZEUS global PDF fits," Journal of High Energy Physics, vol. 2008, no. 1, article 75, 2008.

[5] M. Glück, S. Kretzer, and A. Vogt, "Dynamical QCD predictions for ultrahigh energy neutrino cross sections," Astroparticle Physics, vol. 11, no. 3, pp. 327-334, 1999.

[6] D. K. Choudhury and P. K. Dhar, "An improved analysis of deep inelastic neutrino data with the DGLAP equations at low and high X," Indian Journal of Physics, vol. 81, no. 2, p. 259, 2007.

[7] D. K. Choudhury and P. K. Dhar, "An improved analysis of t-evolution of singlet structure function at low $x$," in Paper Presented at 5th Bilinniel Conference of the Physics Academy of North-East India (PANE), Manipur University, Manipur, India, October, 2010.

[8] "The Ckm matrix and the uniterity triangle," in Proceedings of the Workshop Held at CERN, M. Battaglia, A. J. Buras, P. Gambino, and A. Stocchi, Eds., February 2002.

[9] C. Amsler, M. Doser, M. Antonelli et al., "Review of paticle physics," Physics Letters B, vol. 667, no. 1-5, pp. 1-6, 2008.

[10] P. K. Dhar and D. K. Choudhury, "Cross sections of the neutrino-nucleon interaction at ultra high energy," Journal of Korean Physical Society, vol. 63, no. 8, pp. 1537-1548, 2013.

[11] C. Adloff, V. Andreevx, B. Andrieu et al., "On the rise of the proton structure function $\mathrm{F}_{2}$ towards low $x$," Physics Letters $B$, vol. 520, no. 3-4, pp. 183-190, 2001.

[12] R. Fiore, L. L. Jenkovszky, A. V. Kotikov, F. Paccanoni, A. Papa, and E. Predazzi, "Ultra-high energy neutrino-nucleon interactions," Physical Review D, vol. 68, no. 9, Article ID 093010, 8 pages, 2003.

[13] R. Gandhi, C. Quigg, M. H. Reno, and I. Sarcevic, "Ultrahighenergy neutrino interactions," Astroparticle Physics, vol. 5, no. 2, pp. 81-110, 1996.

[14] A. D. Martin, R. G. Roberts, W. J. Stirling, and R. S. Thorne, "MRST partons generated in a fixed-flavour scheme," Physics Letters B, vol. 636, no. 5, pp. 259-264, 2006.

[15] A. D. Martin, R. G. Roberts, W. J. Stirling, and R. S. Thorne, http://hepdata.cedar.ac.uk/pdf/pdf3.html.

[16] M. H. Reno, "High energy neutrino cross sections," Nuclear Physics B, vol. 143, pp. 407-413, 2005. 

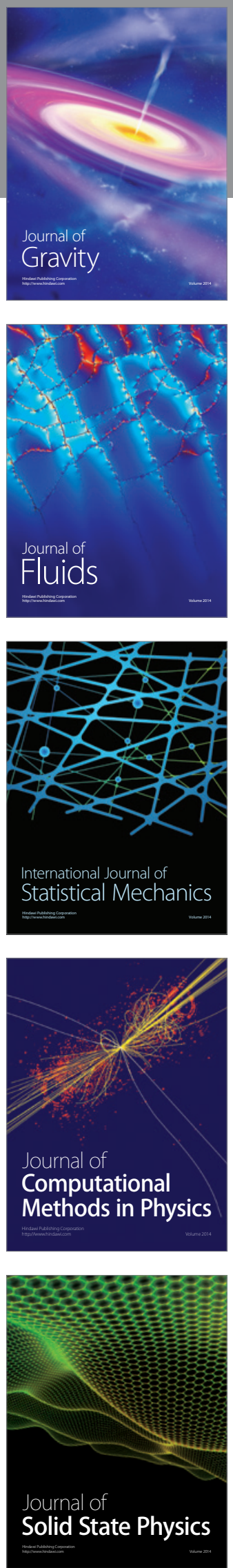

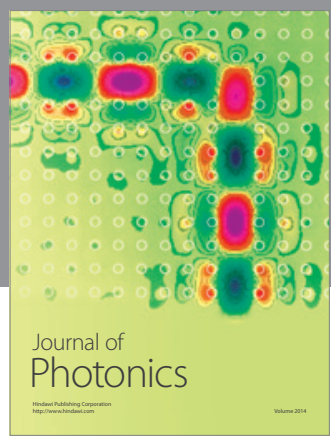

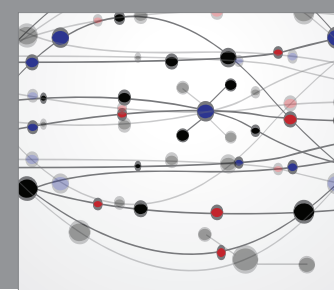

The Scientific World Journal

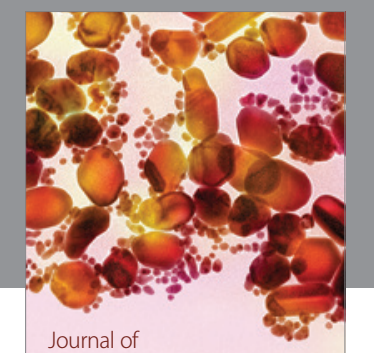

Soft Matter
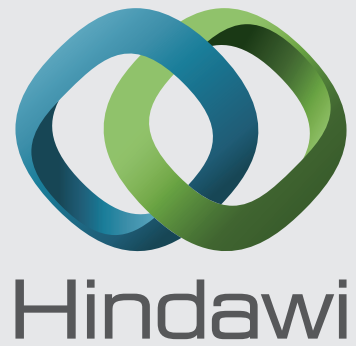

Submit your manuscripts at

http://www.hindawi.com
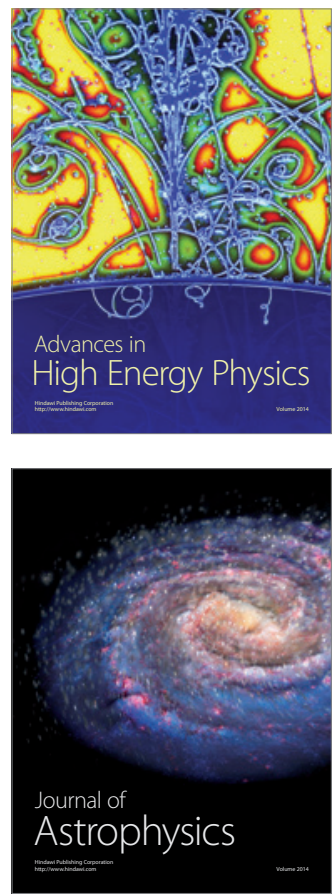
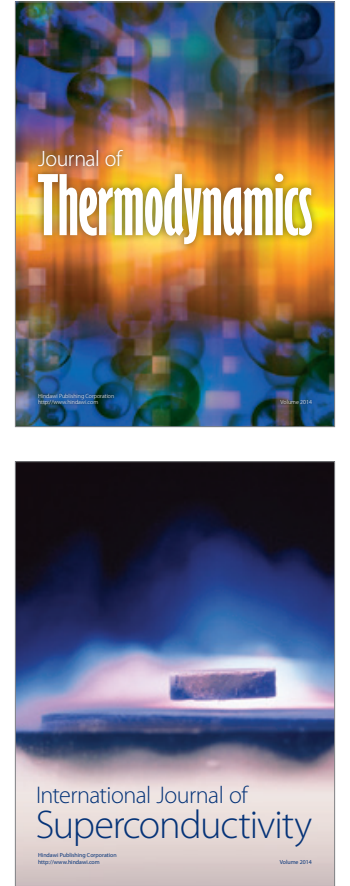
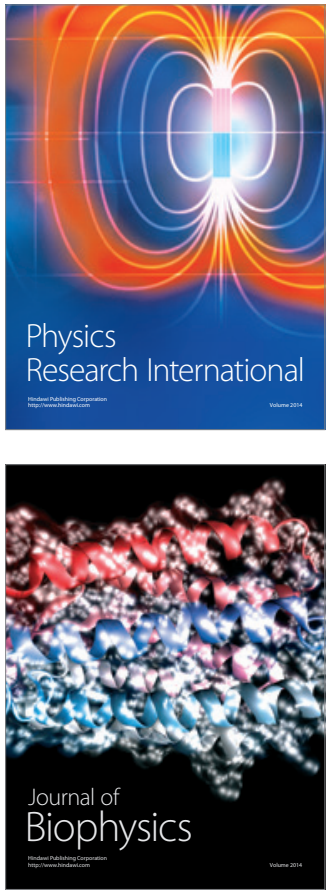
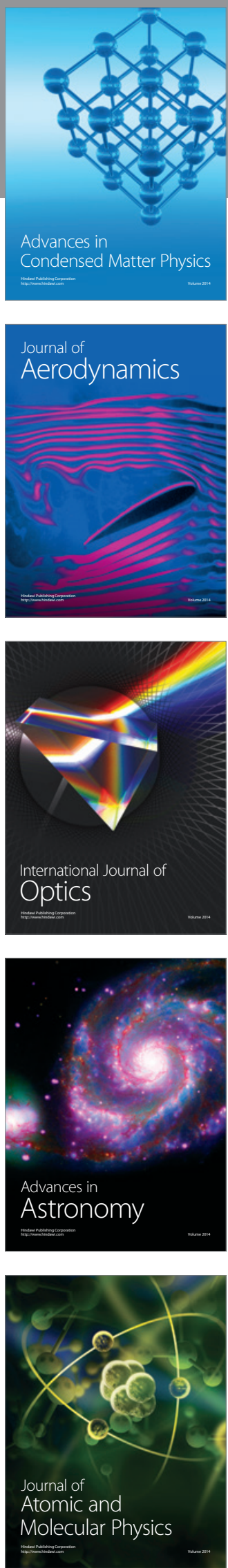